\title{
Aquaporin-1 as an Endothelial Cell Marker in Oral Squamous Cell Carcinoma
}

\author{
Gayatri Nayanar ${ }^{1}$, Mamatha GS Reddy ${ }^{2}$, Elizabeth C Dony ${ }^{3}$
}

\begin{abstract}
Aim and objective: The current study aimed to propose Aquaporin-1 (AQP1) as an endothelial marker and thus explore its role in oral squamous cell carcinoma.

Materials and methods: The study included 30 cases of oral squamous cell carcinoma (OSCC) and 10 normal controls. All the sections were subjected to immunohistochemistry using AQP1 antibody. Images were captured, and quantification of blood vessels was done by assessing the mean microvascular density (MVD) using an image analyzer, and statistical data were computed using one-way analysis of variance test.

Results: The results demonstrated a significant expression of AQP1 in blood vessels of tumor and showed a significant statistical correlation ( $p$ value $<0.05$ ) between mean vascular densities of controls and OSCC cases.

Conclusion: The results have demonstrated a strong immunolocalization of AQP1 in the tumor vasculature, thereby proposing it as a selective marker for the endothelium and suggests its possible involvement in tumor angiogenesis, growth, progression, and prognosis.

Clinical significance: Angiogenesis is a crucial phenomenon in tumor progression. Aquaporin-1 is a proven marker for the endothelial vasculature, and being a functionally efficient protein associated with the progression of tumor through tumor angiogenesis, its blockade can serve in the downregulation of tumor progression.

Keywords: Aquaporin-1, Endothelial marker, Oral squamous cell carcinoma, Tumor angiogenesis.

World Journal of Dentistry (2020): 10.5005/jp-journals-10015-1736
\end{abstract}

\section{INTRODUCTION}

Oral squamous cell carcinoma (OSCC), the most common of the malignant tumors of the oral and maxillofacial regions, accounts for $90 \%$ of all cases. ${ }^{1}$ India has one of the highest incidences of oral cancer and accounts for about $30 \%$ of all new cases annually. ${ }^{2}$ In the past few decades, the therapeutic strategies for OSCC have not shown significant success in the prognosis and overall survival rates. Moreover, the toxicity associated with the presently available systemic therapy is not uncommon. This highlights the need for new biomarkers as novel therapeutic targets.

Tumors require increased nourishment so as to grow and evolve and also to be able to metastasize. ${ }^{3}$ Studies in the past have traced the significant role of angiogenesis in tumor growth and progression. ${ }^{3-5}$ An aberrant angiogenic activity can therefore indicate commencement of a disease process or its advancement. Therefore, an important therapeutic approach for combating this dreadful disease and other angiogenesis-related diseases can certainly be the prohibition of angiogenesis.

Research focusing on specific proteins that control abnormal angiogenesis have gained special interest in the recent times. Such proteins are able to target several angiogenic factors concurrently, thereby increasing the possibility of therapeutic success. Aquaporin-1 (AQP1) is a well-recognized water channel protein facilitating transportation of water and small solutes across the plasma membrane. ${ }^{6}$ It was discovered by Peter Agre in 1992 and was the first discovered water channel protein. ${ }^{7}$ These membrane proteins are a part of the super family of major intrinsic proteins. ${ }^{8}$ Aquaporin-1 is arranged as a tetramer, and AQP1 monomers consists of six tilted a-helical domains $(\mathrm{H} 1-\mathrm{H} 6)$ connected by five loops. ${ }^{6}$ This protein codes for an aquaporin that is involved in the function of transport water, glycerol, gas and ions at a molecular
${ }^{1-3}$ Department of Oral Pathology and Microbiology, Dr DY Patil Dental College and Hospital, Dr DY Patil Vidyapeeth, Pune, Maharashtra, India Corresponding Author: Gayatri Nayanar, Department of Oral Pathology and Microbiology, Dr DY Patil Dental College and Hospital, Dr DY Patil Vidyapeeth, Pune, Maharashtra, India, Phone: +91 9970383646, e-mail: gaya3nayanar@gmail.com

How to cite this article: Nayanar G, Reddy MGS, Dony EC. Aquaporin-1 as an Endothelial Cell Marker in Oral Squamous Cell Carcinoma. World J Dent 2020;11(4):294-298.

Source of support: Nil

Conflict of interest: None

level. To date, 13 Aquaporins (AQPs) have been identified in humans (AQP0-AQP12). ${ }^{9}$ All aquaporins are membrane pores associated with the function of transport of water and several small solutes and only differ in their site of expression. Their physiological expression is seen in the plasma membranes of red blood cells, gastrointestinal tracts, lungs, vascular endothelium, and renal tubules. In addition to the physiological expression in many epithelia and organ systems, novel studies suggest a role for AQPs in the development and progression of different types of cancers. In particular, studies performed on AQP null mice and cell culture models have demonstrated that AQP1 overexpression facilitates cell migration which in turn plays a pivotal role in tumor spreading as well as in tumor angiogenesis. ${ }^{10-12}$ Therefore, this strongly indicates that inhibition of AOP1 will significantly interfere with tumor angiogenesis, thereby affecting its further growth and progression. Also, using it as a marker can facilitate determination of the degree of tumor angiogenesis which can help in assessing the prognosis of the tumor. In the present study, we have proposed AQP1 as an endothelial marker and have thus explored its role in OSCC. 


\section{Materials and Methods}

\section{Tissue Specimens}

Thirty OSCC archival samples of formalin-fixed, paraffinembedded tissue blocks were retrieved from the Department of Oral Pathology and Microbiology, Dr DY Patil Dental College and Hospital, Dr DY Patil Vidyapeeth, Pune, Maharashtra, India. The OSCC cases included comprised of 19 well-differentiated and 11 moderately differentiated cases. Tumor size (T) of all the included cases were considered as part of clinical staging. The included cases were in the age-group of 30-70 years and showed a male predominance ( $\mathrm{M}: \mathrm{F}=2: 1)$. The cases showed anterior, posterior, and anteroposterior mandibular involvement with most common involvement of the posterior mandible. Ten sections of normal buccal mucosa were used as controls and were obtained from voluntary patients who visited the college for tooth extraction upon their consent.

\section{Immunohistochemistry}

Paraffin-embedded tissue blocks of 30 OSCC cases were cut into $4 \mu \mathrm{m}$-thick sections and were subjected to hematoxylin and eosin staining followed by immunohistochemical staining using the immunohistochemistry kit (Dako biotechnology) for AQP1 monoclonal antibody (Biorbyt). The tissue sections of $4 \mu \mathrm{m}$ thickness were deparaffinized and rehydrated in a graded series of alcohol. Endogenous peroxidase activity was blocked with $0.3 \%$ $\mathrm{H}_{2} \mathrm{O}_{2}$ for 30 minutes. The sections were microwaved three times for 5 minutes each in citrate phosphate buffer $(\mathrm{pH}: 6.0)$ for antigen retrieval. The sections were then incubated with protein block serum-free medium for 10 minutes to block nonspecific binding. Monoclonal antibody of AQP1 was applied as primary antibody and incubated at $4^{\circ} \mathrm{C}$ overnight. After washing with phosphatebuffered saline, secondary antibody was applied to the sections which were then incubated for 1 hour in room temperature. Primary antibody was visualized with diaminobenzidine. Sections were counterstained with Mayer's hematoxylin, dehydrated, and mounted.

\section{Quantification of Tumor Vasculature}

Angiogenesis can be measured by light microscopy through the quantification of blood vessels in areas of high vessel density in tissues. Accordingly, the angiogenic activity of the lesion was determined based on the micro-vessel count as described by Maeda et al. The mean microvascular density (MVD) was then calculated for well-differentiated and moderately differentiated cases of OSCC and for the clinical staging of tumor with respect to the T. Blood vessels were considered to be the positive internal controls in the present study.

Three areas showing highest vascularization were identified and scanned initially at low magnification (10x). Later, the three areas showing highest vascularization were identified subjectively by evaluating the histological sections at a higher magnification $(40 \times)$, and the vessels were counted. Images were captured and quantified in an image analyzer. The slides were assessed by two observers to check interobserver variability.

\section{Statistical Analysis}

Data are expressed as mean \pm standard error. Statistically significant differences were computed using one-way analysis of variance test, with the significance level being set at $p$ value $<0.05$.

\section{Results}

The study comprised of 30 cases of OSCC and 10 normal buccal mucosal tissue sections as control. The cases showed a male predominance ( $M: F=2: 1$ ). Half of the population was in the agegroup 30-70 years. Tumors were located most commonly in the mandibular posterior region (alveolobuccal region), with every case being associated with the etiology of tobacco use. Of the 30 cases, 19 cases were well-differentiated OSCC and 11 were moderately differentiated OSCC. The study did not include any cases of poorly differentiated OSCC. Only T factor of TNM tumor staging was considered, as the cases included showed no nodal involvement and metastasis (Table 1).

The mean MVD for OSCC cases was 14.3 and that for controls was 7.69 (Figs 1 and 2). The MVD for well-differentiated OSCC cases was 13.36 and 14.73 for moderately differentiated OSCC cases. The MVD for clinical staging of OSCC with respect to tumor size were $24,14.33,14.83$, and 12.53, respectively, for $\mathrm{T} 1, \mathrm{~T} 2, \mathrm{~T} 3$, and $\mathrm{T} 4$, respectively (Table 2 ).

MVD of OSCC cases and controls (Group I) showed significant results ( $p$ value $=0.001, p$ value $<0.05$ ). Significant results were found when controls and moderately differentiated OSCC cases (Group II) ( $p$ value $=0.01, p$ value $<0.05$ ) and clinical stages T1 and T4 (Group III) were compared ( $p$ value $=0.05, p$ value $<0.05$ ) (Table 3). No significant inter-and intragroup correlation was noted with respect to the histological grades and other clinical stages.

\section{Discusssion}

Tumor growth and metastasis is facilitated by an essential phenomenon, i.e., tumor angiogenesis. Studies have shown significant association of many immunohistochemical endothelial markers with tumor angiogenesis. A potent role of the AQP1 as a tumor angiogenesis inducer has been traced in several studies in the past. Several clinical investigations have explored the upregulated expression of AQP1 as in human astrocytoma and also in rat models of melanoma and breast tumors. ${ }^{10-12}$

Gannon et al. in his study observed physiological expression of AQP1 in the micro-vessel endothelial cells in the rat intestine. ${ }^{13}$ Also, in the circumventricular organs in rat brain, including the choroid

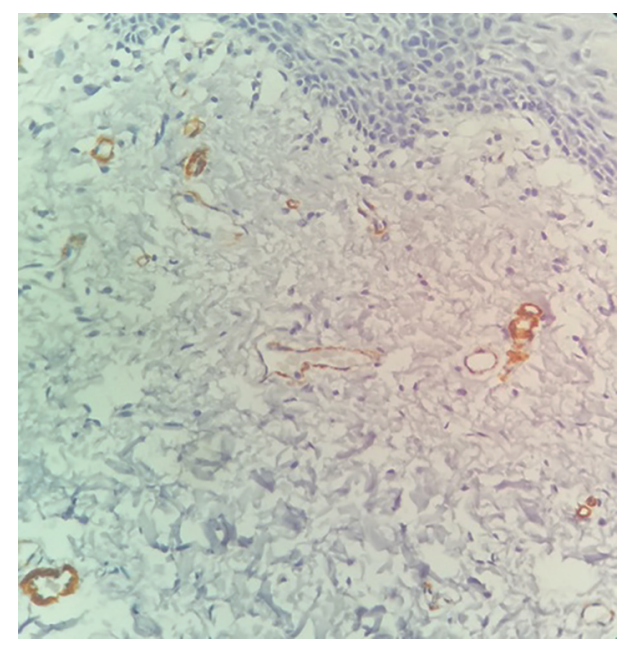

Fig. 1: Photomicrograph showing immunoreactivity of aquaporin-1 in vasculature of normal tissue (Immunohistochemistry, total magnification $\times 400$ ) 


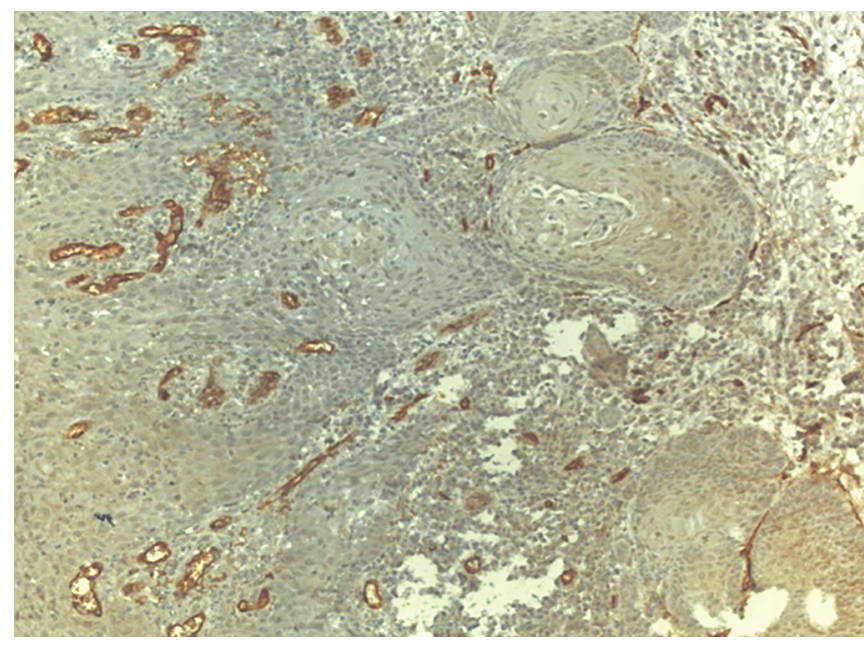

Fig. 2: Photomicrograph showing immunoreactivity of aquaporin-1 in vasculature of well-differentiated oral squamous cell carcinoma showing increased (Immunohistochemistry, total magnification $\times 400$ ) plexus, pineal and medial eminence, subfornical organ, postrema expression of AQP1 was noted by Wilson et al. ${ }^{14}$

Some of these studies have demonstrated the distribution of AQP1 in these transplanted tumors in rats and mice and have found a heterogeneous expression in tumor cells and their vasculature. Luo et al. demonstrated the expression of AQP1 in micro-vessel endothelial cells in human hepatocellular carcinoma. ${ }^{15}$ Stromal endothelial cells in cervical cancer also predominantly expressed AQP1 in a study from China. ${ }^{16}$ In another study by Mobasheri et al., AQP1 was assessed in human tumors of prostate, breast, ovary, colon, and lungs and was seen that its expression was considerably higher in its vascular elements. ${ }^{17}$ An increased degree of AQP1 reactivity was noted by Vacca et al. in the bone marrow microvessels in multiple myeloma patients. ${ }^{18}$ All the above mentioned studies suggest a significant pathological expression of AQP1 in endothelial cell of microvasculature of various tumors.

Association of OSCCs with increased vascularization has been proven in the past studies, supporting the significant role of angiogenesis in its progression, thereby helping in predicting

Table 1: Demographic details and count of blood vessels

\begin{tabular}{|c|c|c|c|c|c|c|}
\hline Sr. No. & Age/Sex & Site & Location & $\begin{array}{l}\text { Histopathological } \\
\text { grading of OSCC }\end{array}$ & TNM staging & Blood vessel $40 \times$ \\
\hline 1 & $60 / F$ & Mandible & Anteroposterior & Well-differentiated & $\mathrm{T} 3$ & 7 \\
\hline 2 & $50 / \mathrm{M}$ & Mandible & Posterior & Well-differentiated & $\mathrm{T} 1$ & 31 \\
\hline 3 & $45 / F$ & Mandible & Anteroposterior & Well-differentiated & $\mathrm{T} 2$ & 23 \\
\hline 4 & $58 / \mathrm{M}$ & Maxilla & Anteroposterior & Well-differentiated & $\mathrm{T} 2$ & 13 \\
\hline 5 & $30 / \mathrm{M}$ & Mandible & Anteroposterior & Well-differentiated & T4 & 7 \\
\hline 6 & $65 / M$ & Mandible & Posterior & Well-differentiated & $\mathrm{T} 2$ & 16 \\
\hline 7 & $50 / \mathrm{M}$ & Mandible & Posterior & Moderately differentiated & T4 & 15 \\
\hline 8 & $55 / F$ & Mandible & Anteroposterior & Well-differentiated & $\mathrm{T} 2$ & 7 \\
\hline 9 & $56 / F$ & Mandible & Posterior & Moderately differentiated & $\mathrm{T} 2$ & 19 \\
\hline 10 & $65 / M$ & Mandible & Posterior & Well-differentiated & $\mathrm{T} 2$ & 16 \\
\hline 11 & $60 / M$ & Tongue & Anteroposterior & Moderately differentiated & T4 & 20 \\
\hline 12 & $55 / F$ & Mandible & Anteroposterior & Well-differentiated & $\mathrm{T} 2$ & 8 \\
\hline 13 & $65 / M$ & Mandible & Anteroposterior & Moderately differentiated & T4 & 12 \\
\hline 14 & $35 / F$ & Tongue & Anteroposterior & Moderately differentiated & T4 & 11 \\
\hline 15 & $54 / \mathrm{M}$ & Mandible & Anteroposterior & Well-differentiated & T3 & 11 \\
\hline 16 & $57 / M$ & Mandible & Anteroposterior & Well-differentiated & T4 & 17 \\
\hline 17 & $45 / \mathrm{M}$ & Mandible & Anteroposterior & Well-differentiated & T4 & 18 \\
\hline 18 & $58 / \mathrm{M}$ & Maxilla & Posterior & Well-differentiated & T4 & 9 \\
\hline 19 & $67 / F$ & Mandible & Anteroposterior & Well-differentiated & $\mathrm{T} 2$ & 13 \\
\hline 20 & $55 / F$ & Maxilla & Anteroposterior & Well-differentiated & T3 & 7 \\
\hline 21 & $50 / F$ & Mandible & Anteroposterior & Moderately differentiated & T4 & 9 \\
\hline 22 & $45 / \mathrm{M}$ & Mandible & Anteroposterior & Moderately differentiated & T4 & 6 \\
\hline 23 & $45 / \mathrm{M}$ & Mandible & Posterior & Moderately differentiated & T3 & 25 \\
\hline 24 & $55 / M$ & Maxilla & Anteroposterior & Well-differentiated & T4 & 11 \\
\hline 25 & $31 / M$ & Mandible & Posterior & Moderately differentiated & T4 & 12 \\
\hline 26 & $43 / M$ & Mandible & Posterior & Moderately differentiated & T3 & 19 \\
\hline 27 & $70 / M$ & Mandible & Posterior & Well-differentiated & T3 & 20 \\
\hline 28 & $57 / M$ & Mandible & Posterior & Well-differentiated & $\mathrm{T} 1$ & 17 \\
\hline 29 & $54 / \mathrm{M}$ & Mandible & Anteroposterior & Moderately differentiated & $\mathrm{T} 2$ & 14 \\
\hline 30 & $65 / F$ & Mandible & Anteroposterior & Well-differentiated & T4 & 16 \\
\hline
\end{tabular}

OSCC, oral squamous cell carcinoma; TNM, tumor, node, and metastasis 
Table 2: Mean microvascular density

\begin{tabular}{ll}
\hline Groups & Mean MVD \\
\hline OSCC cases & 7.69 \\
Controls & 14.3 \\
Well-differentiated OSCC & 13.36 \\
Moderately differentiated OSCC & 14.73 \\
T1 & 24 \\
T2 & 14.33 \\
T3 & 14.83 \\
T4 & 12.53 \\
\hline
\end{tabular}

MVD, microvascular density; OSCC, oral squamous cell carcinoma

Table 3: Statistical significance

\begin{tabular}{ll}
\hline Groups & $p$ value, significance (S) \\
\hline Group I & $0.001, \mathrm{~S}$ \\
Group II & $0.01, \mathrm{~S}$ \\
Group III & $0.05, \mathrm{~S}$ \\
\hline
\end{tabular}

$p$ value < 0.05; Group I: OSCC cases vs controls; Group II: controls vs moderately differentiated cases; Group III: clinical stages T1 vs T4.

its prognosis. ${ }^{3}$ The present study has made an attempt to immunolocalize the blood vessels in OSCC as a method of measuring angiogenesis using AQP1 and thereby proposing it as an endothelial marker. In this study, when comparison was done between the histological grades of OSCC, the results were not statistically significant. This, however, was higher in moderately differentiated OSCC cases compared to the control. Although statistically insignificant, a positive correlation was seen between MVD of histopathological grades of OSCC, with moderately differentiated cases showing higher MVD compared to welldifferentiated cases.

Significant statistical difference was found when comparison was done between clinical stages $\mathrm{T} 1$ and $\mathrm{T} 4$, with $\mathrm{T} 1$ being higher compared to T4. These results were in accordance with the results of the study by Marion-Audibert et al., where it was assessed in pancreatic tumors and was observed to be significantly higher in tumors less than $2 \mathrm{~cm}$ in diameter. The study also suggested MVD to be one of the prognostic factors for identification of risk of metastatic dissemination along with other factors such as size, proliferation index, and evidence of local invasion. ${ }^{19}$

Many endothelial markers have been introduced and studied in the literature for the quantification of microvasculature in various cancers such as CD31, CD34, von Willebrand factor, endoglin, and coagulation factor VIII. ${ }^{15}$ However, to our knowledge, the role of AQP1 as an endothelial marker is yet to be explored, and the current study has made an attempt to demonstrate it in OSCC, making the study a first of its kind. The current study too showed a noted expression of AQP1 in the endothelial cells of vascular structures in OSCC showing agreement with other past studies in different tumors. Expression of AQP1 in endothelia could indicate its probable involvement in tumor angiogenesis. This study has evaluated the role of AQP1 in angiogenesis by assessing it in OSCC. Accordingly, after assessing, a significant increase was noted in the in OSCC cases when compared to the normal controls. The mean MVD considerably correlated with the moderately differentiated grade but not with the clinical staging of OSCC.

In hepatocellular carcinoma, a marked immunohistochemical expression of AQP1 was seen in the membranes of both microvasculature and small vessels. AQP1 demonstrated in micro-vessels along with other small and big vessels in the tumor stroma can also help in assessing neoangiogenesis, thus serving in predicting prognosis and therefore could also probably serve a same role in OSCC. ${ }^{15}$

Although the current study has proposed a possible association of AQP1 in tumor angiogenesis and therefore its progression, it lacks a clear delineation of its precise role in the same. Moreover, the study also presents a small sample size as one of its limitations.

\section{Conclusion}

The results of the current study have demonstrated AQP1 as a selective endothelial marker and has also showed a positive role of AQP1 in angiogenesis by the evidence of a significant increase in mean MVD in higher histological grading of OSCC.

\section{Clinical Significance}

Angiogenesis is a crucial phenomenon in tumor progression. AQP1 is a reportedly strongly expressed protein on the endothelial vasculature, and being a functionally efficiently protein associated with the progression of tumor through tumor angiogenesis, its blockade can serve in the downregulation of tumor progression, and its expression can help assess the severity of the disease serving prognostic purposes.

\section{Ethical Clearance}

Ethical clearance was obtained from Institutional Sub Committee.

\section{ACKnowledgment}

We would like to thank Mrs Hema Choughule for her technical assistance with immunohistochemistry.

\section{References}

1. International Agency for Research on Cancer. The Agency, Lyon 2006.

2. Sankaranarayanan R, Ramadas K, Thomas G, et al. Effect of screening on oral cancer mortality in Kerala, india: a cluster-randomised controlled trial. Lancet 2005;365(9475):1927-1933. DOI: 10.1016/ S0140-6736(05)66658-5.

3. Ascani G, Balercia P, Messi M, et al. Angiogenesis in oral squamous cell carcinoma. Acta Otorhinolaryngol Ital 2005;25(1):13-17.

4. Jaeger TM, Weidner N, Chew K, et al. Tumor angiogenesis correlates with lymph node metastases in invasive bladder cancer. J Urol 1995;154(1):69-71. DOI: 10.1016/S0022-5347(01)67230-6.

5. Sakamoto K, Maeda S, Hikiba Y, et al. Constitutive NF-KB activation in colorectal carcinoma plays a key role in angiogenesis, promoting tumor growth. Clin Cancer Res 2009;15(7):2248-2258. DOI: 10.1158/1078-0432.CCR-08-1383.

6. Wang J, Feng L, Zhu Z, et al. Aquaporins as diagnostic and therapeutic targets in cancer: How far we are? J Transl Med 2015;13:96. DOI: 10.1186/s12967-015-0439-7.

7. Agre PE, Preston GM, Smith BL, et al. Aquaporin CHIP: the archetypal molecular water channel. Am J Physiol 1993;265(4):F463-F476. DOI: 10.1152/ajprenal.1993.265.4.F463.

8. Benga G. On the definition, nomenclature and classification of water channel proteins (aquaporins and relatives). Mol Aspects Med 2012;33(5-6):514-517. DOI: 10.1016/j.mam.2012.04.003.

9. Nico B, Ribatti D. Role of aquaporins in cell migration and edema formation in human brain tumors. Exp Cell Res 2011;317(17):23912396. DOI: 10.1016/j.yexcr.2011.07.006.

10. Esteva-Font C, Jin BJ, Verkman AS. Aquaporin-1 gene deletion reduces breast tumor growth and lung metastasis in tumor-producing MMTVPyVT mice. FASEB J 2014;28(3):1446-1453. DOI: 10.1096/fj.13-245621. 
11. Nicchia GP, Stigliano C, Sparaneo A, et al. Inhibition of Aquaporin-1 dependent angiogenesis impairs tumour growth in a mouse model of melanoma. J Mol Med (Berl) 2013;91(5):613-623. DOI: 10.1007/ s00109-012-0977-x.

12. El Hindy N, Bankfalvi A, Herring A, et al. Correlation of Aquaporin-1 water channel protein expression with tumor angiogenesis in human astrocytoma. Anticancer Res 2013;33(2):609-613.

13. Gannon BJ, Carati CJ. Endothelial distribution of the membrane water channel molecule aquaporin-1: implications for tissue and lymph fluid physiology? Lymphat Res Biol 2003;1(1):55-66. DOI: 10.1089/15396850360495709.

14. Wilson AJ, Carati CJ, Gannon BJ, et al. Aquaporin-1 in blood vessels of rat circumventricular organs. Cell Tissue Res 2010;340(1):159-168. DOI: 10.1007/s00441-010-0927-2.

15. Luo LM, Xia H, Shi R, et al. The association between aquaporin-1 expression, microvessel density and the clinicopathological features of hepatocellular carcinoma. Oncol Lett 2017;14(6):7077-7084. DOI: 10.3892/ol.2017.7106.
16. Shi YH, Chen R, Talafu T, et al. Significance and expression of aquaporin $1,3,8$ in cervical carcinoma in Xinjiang uygur women of china. Asian Pac J Cancer Prev 2012;13(5):1971-1975. DOI: 10.7314/APJCP.2012. 13.5.1971.

17. Mobasheri A, Airley R, Hewitt SM, et al. Heterogeneous expression of the aquaporin 1 (AQP1) water channel in tumors of the prostate, breast, ovary, colon and lung: a study using high density multiple human tumor tissue microarrays. Int J Oncol 2005;26(5):1149-1158. DOI: 10.3892/ijo.26.5.1149.

18. Vacca A, Frigeri A, Ribatti $D$, et al. Microvessel overexpression of aquaporin 1 parallels bone marrow angiogenesis in patients with active multiple myeloma. $\mathrm{Br} J$ Haematol 2001;113(2):415-421. DOI: 10.1046/j.1365-2141.2001.02738.x.

19. Marion-Audibert AM, Barel C, Gouysse G, et al. Low microvessel density is an unfavorable histoprognostic factor in pancreatic endocrine tumors. Gastroenterology 2003;125(4):1094-1104. DOI: 10.1016/S0016-5085(03)01198-3. 